\title{
海萤荧光素衍生物发光反应关键步骤的理论研究
}

\author{
孙颖任爱民* 闵春刚邹陆一 任雪峰 \\ (吉林大学理论化学研究所, 理论化学计算国家重点实验室, 长春 130023)
}

\begin{abstract}
摘要：用不同取代基对化学发光物质 6-芳基-2-甲基咪唑[1,2- $\alpha$ 吡嗪-3-(7H)酮环(海萤发光的类似物)的芳基位 进行取代, 形成系列海茧苂光素类似物 MIPa-MIPd; 并采用 B3LYP/6-311+G(d,p)方法, 通过电子抽取能(EEP)的 计算和自然电荷布居分析(NPA), 研究了在气相、二甲亚砜(DMSO)和二甘醇二甲醚(DG)中海萤发光的类似物从 阴离子变化到自由基过程中取代基的作用. 结果表明: 在这个过程中, 吲梠在 DG 中作为取代基(MIPb)时的 EEP 最小, 电荷变化最大, 说明这种取代基有利于反应的进行.
\end{abstract}

关键词：密度泛函理论；海萤苂光素；化学发光；电子抽取能；自然电荷布居分析 中图分类号：0641

\section{Theoretical Investigation of the Key Reaction for the Chemiluminescence of Cypridina Luciferin Analogues}

\author{
SUN Ying REN Ai-Min* MIN Chun-Gang ZOU Lu-Yi REN Xue-Feng \\ (State Key Laboratory of Theoretical and Computational Chemistry, Institute of Theoretical Chemistry, Jilin University, \\ Changchun 130023, P. R. China)
}

\begin{abstract}
A series of 6-aryl-2-methylimidazo[1,2- $\alpha$ ]pyrazin-3-(7H)-ketone based derivatives MIPa-MIPd (cypridina luciferin analogues) were investigated using the B3LYP/6-311+G( $d, p)$ method, and we modeled a 6-site replacement by different substituents. The effect of different substitutions on cypridina luciferin analogues in their transition from anions to free radicals was studied by electron extraction potentials (EEP) and natural charge population analysis (NPA) in gas phase, dimethyl sulfoxide (DMSO), and diglyme (DG). The calculated results indicated that MIPb (3-indolyl as the substituent) had a lower EEP and a larger natural charge population change occurred at the MIP moiety in DG when transitioning from the anion to its free radical than in the other solvents. We also showed that 3-indolyl, as a substituent, accelerated the luminescent reaction of MIP.
\end{abstract}

Key Words: Density functional theory; Cypridina luciferin; Chemiluminescence;

Electron extraction potential; Natural charge population analysis

海茧由于高的发光效率而成为研究热点. 海茧 把苂光素和苂光素酶喷射到海水中产生蓝色苂光 (发光效率 0.3), 海萤荧光素的晶体结构在 1957 年 由 Shimomura 等 ${ }^{[1]}$ 测出. 由于生物发光的灵敏度很 高, 所以海苂苂光素的研究对生物分子的快速敏感
检测和对功能活性分子以及细胞的活动规律进行 原位跟踪观察发挥着非常重要的作用 ${ }^{[2]}$. 海菼苂光 素在生物医学研究 ${ }^{[3]}$ 临床诊断、食品 ${ }^{[4]}$ 以及环境检 测 ${ }^{[5-6]}$ 等方面的应用也越来越广泛, 相关产品也逐步 实现产业化和规模化 ${ }^{[7]}$. 并且海萤苂光素及其菼光

Received: June 7, 2010; Revised: July 17, 2010; Published on Web: September 3, 2010.

*Corresponding author. Email: aimin_ren@yahoo.com; Tel: +86-431-88498567.

The project was supported by the National Natural Science Foundation of China (20673045, 20973078), Start Fund for Returned Overseas People, China ((2008)890), and Major State Basic Research Development Program of China (973) (2002CBN613406).

国家自然科学基金(20673045, 20973078), 留学回国人员启动基金(外交司留(2008)890 号)和国家重点基础研究发展计划项目(973) (2002CBN613406)资助 
素酶都很稳定, 可保存多年, 这可用于某些特殊情况 下的照明 ${ }^{[8]}$. 但是海茧苂光素高效率的发光问题至 今还没有清楚的理论描述. 海萤生物发光通常的反 应机理是海萤在体内苂光素酶和氧气等的作用下, 发光分子(通常称为苂光素)被氧化成激发态, 在退 激的过程中发出可见光, 所以生物发光本质也是化 学发光, 不同之处在于, 在生物发光中激发态的形成 往往伴随酶的作用 ${ }^{[7,9]} .1966$ 年研究者发现, 一个咪 唑并吡嗪酮环的存在是发光反应的核心, 另外海萤 苂光素的同系物也被合成出来, 并用来研究海萤生 物发光的机理 ${ }^{[9-13]}$. Takahashi 等 ${ }^{[14]}$ 对海茧生物发光 的研究表明, 咪唑并吡嗪酮环的阴离子 $\left(\mathrm{MIP}^{-}\right)$和与 氧气反应生成咪唑并吡嗪酮环的自由基(MIP•)的过 程(如示意图 1 所示)对整个发光反应起到了关键的 作用, 并且决定了反应的速率 ${ }^{[1-15]}$. 本文以海萤苂光 素活性中心分子结构咪唑并吡嗪酮环为核心, 在 C6 位上用不同的芳基取代(见图 1), 用密度泛函理论 $(\mathrm{DFT})^{[16-17]}$, B3LYP 方法 ${ }^{[18-20]}$ 结合 $6-311+\mathrm{G}(d, p)$ 基组 从理论上研究这些海茧苂光素衍生物的苂光活性中 心分子的电子结构, 从而从分子水平上理解 C6 位 上取代基的变化对该发光反应决速步骤的影响.

\section{1 理论方法}

密度泛函理论研究化学发光现象的可靠性已得 到广泛证实 ${ }^{[21-22]}$. 本文运用 Gaussian 03 程序包 ${ }^{[23]}$, 采<smiles>CCC(C)c1nc2c(CCCNC(=N)N)[nH]c(-c3c[nH]c4ccccc34)cn-2c1=O</smiles><smiles>Cc1ccc(N(C)C)cc1</smiles>

图 1 6-芳基-2-甲基咪唑 $[1,2-\alpha]$ 吡嗪-3-(7H)酮环和海茧结构 Fig.1 Structures of 6-aryl-2-methylimidazo[1,2- $\alpha$ ] pyrazin-3-(7H)-ketone ring and cypridina luciferin

用密度泛函理论 DFT 中的 B3LYP 泛函方法对类海 茧苂光素发光过程中关键步骤(示意图 1 中的反应

(1)

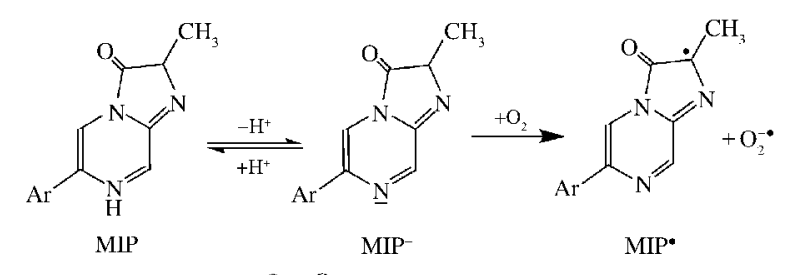

(2)

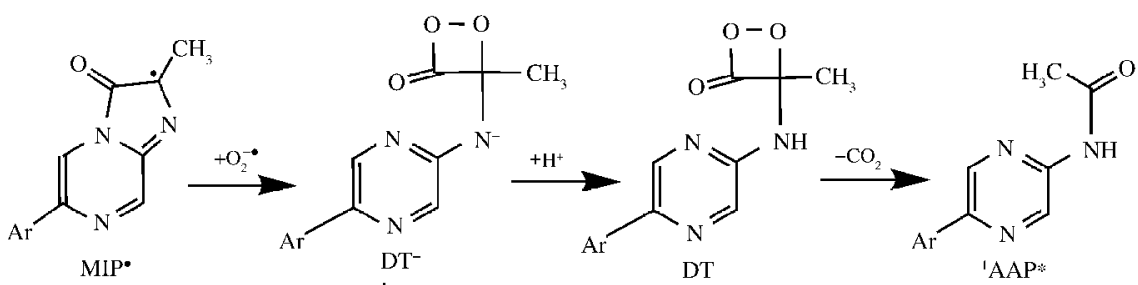

$\downarrow-\mathrm{CO}_{2}$<smiles>CC(=O)Nc1cnc(I)cn1</smiles>

示意图 1 MIP 分子化学发光反应机理

Scheme 1 Chemiluminescent reaction mechanism of MIP 
式(1))涉及的阴离子、自由基的结构进行了优化, 考 虑所研究分子 6-芳基-2-甲基咪唑 [1,2- $\alpha$ 吡嗪-3-(7H) 酮环上 7 位 $\mathrm{H}$ 原子的重要性和相应自由基的活泼 性, 计算中采用了 $6-311+\mathrm{G}(d, p)$ 基组. 文中对 6-芳 基-2-甲基咪唑 [1,2- $\alpha$ ]吡嗪-3-(7H)酮环(海萤发光的 相似物)芳基位不同取代基分子(MIP)从阴离子到自 由基的变化过程(示意图 1 中的反应式(1))的电子 抽取能(EEP)和自然电荷布居进行了计算研究. 在 这里电子抽取能(EEP)定义为阴离子失去一个电子 形成自由基所需的垂直电离能. 运用自然键轨道 $(\mathrm{NBO})$ 分析程序得到所计算分子中的原子自然电荷 布居, 分析各种分子内、分子间电荷变化. 溶剂作用 选用极化连续模型 $(\mathrm{PCM})^{[24]}$ 来模拟. 溶剂二甲亚枫 (DMSO) 和二甘醇二甲醚(DG)的相对介电常数分别 采用 46.7 和 6.25 .

\section{2 结果与讨论}

本文研究的化学发光反应过程(见示意图 1)即 MIP 在碱性溶剂作用下失去 $\mathrm{H}^{+}$离子形成 $\mathrm{MIP}^{-}$, $\mathrm{MIP}^{-}$在氧气等的作用下, 发生电子转移, 即 $\mathrm{O}_{2}$ 抽
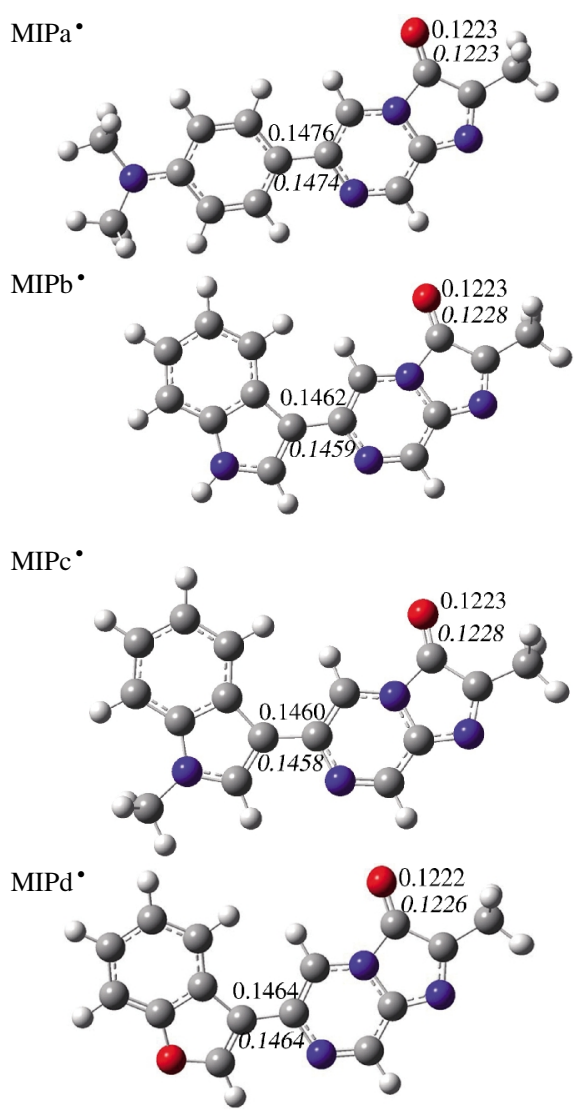

图 2 MIP 阴离子和自由基的优化结构

Fig.2 Optimized structures for the anions and free radicals of MIP

The $\mathrm{C}=\mathrm{C}$ and $\mathrm{C}=\mathrm{O}$ bond lengths (in $\mathrm{nm}$ ) with Roman and italic refer to those in gas phase and $\mathrm{DG}$, respectively. MIPd.

\section{1 分子几何优化} 示.
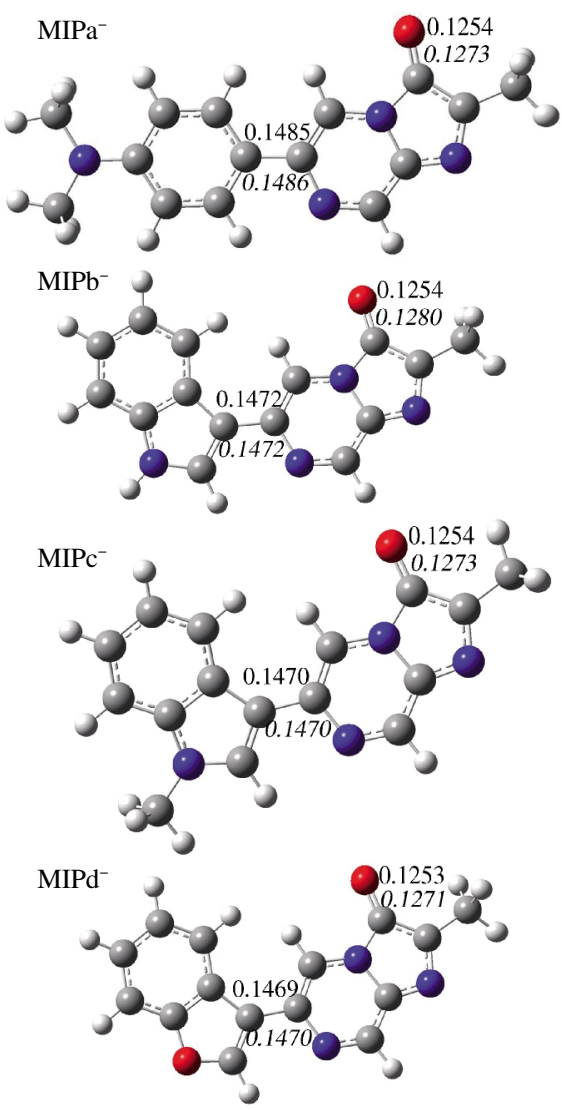

取了一个电子形成 $\mathrm{O}_{2}^{-}$, 使阴离子 $\mathrm{MIP}^{-}$变成自由基 $\mathrm{MIP}^{\bullet}$, 它们继续作用产生了发光分子(DT), 发光分子 被氧化成激发态 $\left({ }^{1} \mathrm{AAP}{ }^{*}\right),{ }^{1} \mathrm{AAP}^{*}$ 在退激的过程中发 出可见光. 其中 $\mathrm{MIP}^{-} \rightarrow \mathrm{MIP}^{\bullet}$ 过程为整个发光反应 的决速步骤 ${ }^{[14]}$. 对这个决速步骤有影响的因素除了 骨架分子结构咪唑并吡嗪酮环外, 取代基的影响是 值得研究的. 已有的报道结果 ${ }^{[2-13]}$ 显示: 在 C6 位上 给电子基团取代味唑并吡嗪酮环有较好的苂光效 率, 所以我们选择了三种给电子基团(如图 1 所示的 $\mathrm{a}-\mathrm{c}$ )和一种吸电子基团(d)对咪唑并吡嗪中心环的 C6 位进行取代, 形成四种反应物 MIPa, MIPb, MIPc,

示意图 1 给出了 $\mathrm{MIP} \rightarrow \mathrm{MIP}^{-} \rightarrow \mathrm{MIP}$ ・变化过 程 ${ }^{[14-15,25-27]}$ 的示意图, 图 1 给出了 C6 位上不同芳基 取代的 MIP 衍生物的具体分子结构, 带有不同取代 基的 MIP 分子阴离子和自由基优化结构如图 2 所

把不同取代基取代咪唑并吡嗪酩化物的中性自 由基分子和阴离子作对比, 结果表明: 在气相中, 给 
表 1 MIPa-MIPd 阴离子和自由基的 $\mathrm{C}-\mathrm{C} 6 、 \mathrm{C}=\mathrm{O}$ 键长及其差值

Table $1 \mathrm{C}-\mathrm{C} 6, \mathrm{C}=\mathrm{O}$ bond lengths and bond length differences of the anions and free radicals for compounds MIPa-MIPd

\begin{tabular}{|c|c|c|c|c|c|c|c|c|}
\hline & \multicolumn{3}{|c|}{ Gas } & \multirow[b]{2}{*}{$\Delta r_{\mathrm{C}=d} / \mathrm{nm}$} & \multicolumn{3}{|c|}{ DG } & \multirow[b]{2}{*}{$\Delta r_{\mathrm{C}=0} / \mathrm{nm}$} \\
\hline & $r_{\mathrm{C}-\mathrm{Cd}} / \mathrm{nm}$ & $\Delta r_{\mathrm{C}-\mathrm{Cd}} / \mathrm{nm}$ & $r_{\mathrm{C}=\mathrm{d}} / \mathrm{nm}$ & & $r_{\mathrm{C}-\mathrm{Cd}} / \mathrm{nm}$ & $\Delta r_{\mathrm{C}-\mathrm{Cd}} / \mathrm{nm}$ & $r_{\mathrm{C}=} \mathrm{d} / \mathrm{nm}$ & \\
\hline $\mathrm{MIPa}^{\bullet}$ & 0.1476 & 0.0009 & 0.1223 & 0.0031 & 0.1474 & 0.0012 & 0.1223 & 0.0050 \\
\hline $\mathrm{MIPa}^{-}$ & 0.1485 & & 0.1254 & & 0.1486 & & 0.1273 & \\
\hline $\mathrm{MIPb}^{\bullet}$ & 0.1462 & 0.0010 & 0.1223 & 0.0031 & 0.1459 & 0.0013 & 0.1228 & 0.0052 \\
\hline $\mathrm{MIPb}^{-}$ & 0.1472 & & 0.1254 & & 0.1472 & & 0.1280 & \\
\hline $\mathrm{MIPc}^{\bullet}$ & 0.1460 & 0.0010 & 0.1223 & 0.0031 & 0.1458 & 0.0012 & 0.1228 & 0.0045 \\
\hline $\mathrm{MIPc}^{-}$ & 0.1470 & & 0.1254 & & 0.1470 & & 0.1273 & \\
\hline MIPd • & 0.1464 & 0.0005 & 0.1222 & 0.0031 & 0.1464 & 0.0006 & 0.1226 & 0.0045 \\
\hline $\mathrm{MIPd}^{-}$ & 0.1469 & & 0.1253 & & 0.1470 & & 0.1271 & \\
\hline
\end{tabular}

$\Delta r$ : bond length difference between the anions and free radicals

受电子基团与 MIP 间基本上保持在同一平面(二面 角在 $179.15^{\circ}-179.90^{\circ}$ 之间, 而吸电子基团为 $173.85^{\circ}$ ), 取代基与 MIP 连接处的 C- $\mathrm{C} 6$ 键长有明显变化. 有研究表明 ${ }^{[14]}$, 当咪唑并吡嗪酮环在溶剂 DG 中时, 其发光效率接近于海萤生物体本身, 所以我们在前 面优化的几何构型下, 采用 PCM 模型在 DG 溶剂中 再次优化其构型. 部分优化结果如表 1 所示, 结果表 明: 在 MIP-变为 $\mathrm{MIP}$ ・时吸电子取代基团与咪唑并 吡嗪酮环的角度发生的变化相对给电子基团偏大, 因而吸电子基团取代时共轭减弱. 阴离子与自由基 中的 $\mathrm{C}-\mathrm{C} 6$ 键长差值的关系是 $\mathrm{MIPb}=\mathrm{MIPc} \approx$ $\mathrm{MIPa}>\mathrm{MIPd}$, 说明取代基为给电子性质时, 从阴离子 到自由基时 $\mathrm{C}-\mathrm{C} 6$ 键长变化比较大并且都缩短了 (气相中缩短了 $0.0009-0.0010 \mathrm{~nm}, \mathrm{DG}$ 中缩短了 0.0012-0.0013 nm), 表明此时的自由基相对阴离子 来说, 取代基与咪唑并吡嗪酮环间共轭加强, 而由 吸电子基取代时 $\mathrm{C}$ - $\mathrm{C} 6$ 键长也缩短了, 但是幅度比 较小 (气相中缩短了 $0.0005 \mathrm{~nm}, \mathrm{DG}$ 中缩短了 0.0006 $\mathrm{nm})$. 这样无论在气相还是溶剂中都呈现一个规律: 具有给电子基的阴离子 $\mathrm{a}, \mathrm{b}, \mathrm{c}$ 相对吸电子基的阴离 子 $\mathrm{d}$ 键长变化大, 给电子基团与中心结构的 $\pi$ 共轭 相互作用加强, 并且在溶剂中 C-C6 键长变化幅度 都大于在气相中, 说明溶剂增强了给电子基团与中 心结构片段之间的相互作用.

\section{2 电子抽取能的计算}

由 Takahashi 等 ${ }^{[14]}$ 的研究可知, 咪唑并吡嗪酮 环的阴离子 $\left(\mathrm{MIP}^{-}\right)$与氧气反应生成咪唑并吡嗪酮 环的自由基(MIP')的过程(如示意图 1 所示)对整个 发光反应起到了关键的作用, 并且决定了反应的速 率 ${ }^{[15]}$. 电子抽取能 EEP 可评估一个分子失去电子的 能力, EEP 越小说明失去电子时需要的能量越小, 即
越容易失去电子. 对于海茧衍生物苂光过程、性质和 苂光效率来说, 除了发光体本身分子结构起决定作 用外, 外界环境如溶剂的影响也不容忽视. 已有实 验报道 ${ }^{[27-28]}$ 表明在非质子溶剂中海萤类似物的苂光 效率较高, 所以, 我们选择了在 DMSO 和 DG 非质 子溶剂中及气相中对 MIPa-MIPd 分子进行深人的 理论研究. 首先我们对反应物 $\mathrm{MIPa}^{-}-\mathrm{MIPd}^{-}$的 HOMO-LUMO 能隙做了分析, 结果列于表 2. 结果表 明, 它们的能隙在气相和 DMSO 中的顺序为 $\mathrm{MIPa}^{-}<$ $\mathrm{MIPd}^{-}<\mathrm{MIPc}^{-}<\mathrm{MIPb}^{-}$, 在溶剂 DG 中变为 $\mathrm{MIPa}^{-}<$ $\mathrm{MIPb}^{-}<\mathrm{MIPc}^{-}<\mathrm{MIPd}^{-}$, 显然 $\mathrm{MIPb}^{-}$在 DG 中的动力学 稳定性降低, 说明在类海茧环境 DG 中 $\mathrm{MIPb}^{-}$在整 个发光反应的决速步骤中优先于其他取代物 $\mathrm{MIPc}^{-}-\mathrm{MIPd}^{-}$.

其次分别计算了 MIPa-MIPd 分子的电子抽取 能(表 3), 通过表 3 可知: 无论是在气相中还是两种 溶剂(DMSO 和 DG)中, 给电子基取代物 MIPa-MIPc 的 EEP 总小于吸电子基取代物 MIPd 的, EEP 越小, 反应越容易进行. 表 3 结果说明给电子基团作为取 代基时更有助于反应快速进行. 在 DMSO 中的 EEP 总体小于在 DG 溶剂中的 EEP, 说明海萤衍生物的 化学发光反应决速步骤(1)在 DMSO 中比在 DG 中

\section{表 2 反应物 $\mathrm{MIPa}^{-}-\mathrm{MIPd}^{-}$在不同介质中的 HOMO- LUMO 能隙 $\left(\Delta \boldsymbol{E}_{\mathrm{HL}}\right)$}

Table 2 HOMO-LUMO energy gaps $\left(\Delta E_{\mathrm{HL}}\right)$ for compounds $\mathrm{MIPa}^{-}-\mathrm{MIPd}^{-}$in different media

\begin{tabular}{cccc}
\hline & $\frac{\Delta E_{\mathrm{H}-\mathrm{L}}(\mathrm{gas})}{\left(\mathrm{kJ} \cdot \mathrm{mol}^{-1}\right)}$ & $\frac{\Delta E_{\mathrm{H}-\mathrm{L}}(\mathrm{DMSO})}{\left(\mathrm{kJ} \cdot \mathrm{mol}^{-1}\right)}$ & $\frac{\Delta E_{\mathrm{H}-\mathrm{L}}(\mathrm{DG})}{\left(\mathrm{kJ} \cdot \mathrm{mol}^{-1}\right)}$ \\
\hline $\mathrm{MIPa}^{-}$ & 214.13 & 320.98 & 316.86 \\
$\mathrm{MIPb}^{-}$ & 231.14 & 326.07 & 321.72 \\
$\mathrm{MIPc}^{-}$ & 228.30 & 325.86 & 324.68 \\
$\mathrm{MIPd}^{-}$ & 225.65 & 324.18 & 325.60 \\
\hline
\end{tabular}


表 3 MIPa-MIPd 分子在不同介质中的电子抽取能

(EEP)

Table 3 Electron extraction potentials (EEP) for compounds MIPa-MIPd in different media

\begin{tabular}{cccc}
\hline & $\frac{\mathrm{EEP}(\mathrm{gas})}{\left(\mathrm{kJ} \cdot \mathrm{mol}^{-1}\right)}$ & $\frac{\mathrm{EEP}(\mathrm{DMSO})}{\left(\mathrm{kJ} \cdot \mathrm{mol}^{-1}\right)}$ & $\frac{\mathrm{EEP}(\mathrm{DG})}{\left(\mathrm{kJ} \cdot \mathrm{mol}^{-1}\right)}$ \\
\hline $\mathrm{MIPa}$ & 214.49 & 407.26 & 421.90 \\
$\mathrm{MIPb}$ & 219.58 & 408.97 & 406.57 \\
$\mathrm{MIPc}$ & 218.43 & 410.16 & 426.89 \\
$\mathrm{MIPd}$ & 239.43 & 419.13 & 428.47 \\
\hline
\end{tabular}

更快速, 这点与实验报道 ${ }^{[25]}$ 相一致. 但在 DG 中, 对 不同取代基化合物的 EEP 比较发现, 吲哚(b)取代物

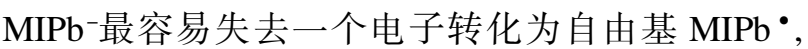
即 EEP 最小, 反应较其他取代物更易进行, 从而从 理论上表明在类海茧环境 DG 下整个发光反应的决 速步骤中吲哚取代时优先于其他取代 MIP, 并且 $b$ 作为 MIP 分子的取代基时更接近海萤的原型 ${ }^{[29-31]}$, 因此这是海萤在 DG 中发光效率比较高 ${ }^{[27-28]}$ 的主要 原因之一.

为了验证在不同溶剂中, 对于反应过程 $\mathrm{MIP}^{-} \rightarrow$ MIP•, 咪唑并吡嗪酮环 C6 位上不同取代基 $\mathrm{Ar}$ 对决 速步骤(1)的贡献作用, 我们还比较了阴离子和自由 基在各自最优构型下的能量差, 结果列于表 4. 从表 4 中可以看出: 取代基为 $\mathrm{a}, \mathrm{b}, \mathrm{c}$ 时的咪唑并吡嗪酮 环的 $\Delta E$ 值一般都小于 $\mathrm{d}$, 这说明给体取代时 $\mathrm{MIP}^{-}$ 到 MIP・这个过程中更容易进行. 在 DMSO 中 $\mathrm{a}, \mathrm{b}, \mathrm{c}$ 取代物的 $\Delta E$ 值总体小于在 DG 中的 $\Delta E$, 并且在 DG 中, 吲哚取代(b)时能量差 $\Delta E$ 最小. 这些结果与 上面 EEP 计算结果一致, 说明 MIP 自由基的弛豫 能在非质子化溶剂中较小, 可忽略, 用 EEP 值可以 判断不同取代基对这个反应的影响.

\section{3 前线轨道及自然电荷布居分析}

首先对四种取代基参与前线轨道的情况加以分 析, 所研究分子在气相和两种溶剂中的前线轨道用 Gaussview 软件绘出并示于图 3 中.

如图 3 所示, 从 LUMO 轨道看, 各种取代基的 阴离子, 其 LUMO 轨道在有溶剂和无溶剂参与时显

表 4 MIPa-MIPd 阴离子和自由基最优构型下的能量差 Table 4 Energy differences between the optimized anions and free radicals for compounds MIPa-MIPd

\begin{tabular}{cccc}
\hline & $\Delta E(\mathrm{gas}) /\left(\mathrm{kJ} \cdot \mathrm{mol}^{-1}\right)$ & $\Delta E(\mathrm{DMSO}) /\left(\mathrm{kJ} \cdot \mathrm{mol}^{-1}\right)$ & $\Delta E(\mathrm{DG}) /\left(\mathrm{kJ} \cdot \mathrm{mol}^{-1}\right)$ \\
\hline MIPa & 199.27 & 391.18 & 405.36 \\
MIPb & 204.78 & 392.50 & 362.04 \\
MIPc & 202.53 & 392.76 & 409.30 \\
MIPd & 223.16 & 402.21 & 417.18 \\
\hline
\end{tabular}

示出特别明显的差别, LUMO 在气相中基本由取代 基组成, 但在 DMSO 和 DG 中则完全由核结构 MIP 组成. 而自由基分子的 LUMO 组成受溶剂影响不 大, 有无溶剂存在都完全由核结构 MIP 组成. 由 $\mathrm{HOMO}$ 轨道看, 气相中各种取代基参与轨道构成较 小, 只有在溶剂协助下, 取代基才参与最高占据轨道 的组成中. $a, b, c$ 三种给电子取代基在自由基分子 的 HOMO 中所占成分远远大于吸电子取代基 $d$, 显 然吸电子的苯唑呋喃基在 $\mathrm{MIP}^{-} \rightarrow$ MIP ${ }^{-}$过程的活化 作用不如其他三个给电子取代基. 对于三个给电子 基的活化自由基作用从轨道分布图上无法区分, 需 要进行电荷布居定量分析进行比较.

利用自然键轨道(NBO)程序计算了 $M I P a 、 M I P b 、$ MIPc 分子中的原子布居数, 采用自然电荷布居分析 (NPA)法分析每一个原子的电荷, 从而得到分子片 段的电荷分布. 分别对海茧核心结构的阴离子和自 由基做了电荷布居分析, 目的是研究 $\mathrm{MIP}^{-} \rightarrow \mathrm{MIP}^{*}$ 过程电荷转移的情况.

根据前面的计算, 我们知道在 $\mathrm{DG}$ 中, 在 $\mathrm{MIP}^{-}$ 到 MIP•这个过程中当吲哚(b)作为咪唑并吡嗪酮 环 C6 位上的取代基时的 EEP 最小, 其电子转移能 力最强, 在整个发光反应的决速步骤中起到了关键 作用. 在 DG 中咪唑并吡嗪酮环上 C6 位上的取代 基是怎样影响中心环的电荷分布呢? 我们对其进 行了 $\mathrm{NBO}$ 计算分析, 其结果示于图 4 .

其中的片段电荷是与取代芳基连接后海茧荧光 素母体部分的电荷量. 由图 4 可知, $\mathrm{MIPa}^{-}-\mathrm{MIPc}^{-}$在 $\mathrm{DG}$ 中一个负电荷主要分布在中心环的三个 $\mathrm{N}$ 原 子、酮基氧和甲基 $\mathrm{C}$ 上. 离取代基最远端的 $\mathrm{N} 1$ 、酮 基 $\mathrm{O}$ 上聚集的电荷在 $\mathrm{b}$ 取代 $\left(\mathrm{MIPb}^{-}\right)$时最多. $\mathrm{b}$ 取代 时, 甲基 $\mathrm{C}$ 原子及酮基 $\mathrm{O}$ 上收集负电荷最多, 失去 一个负电荷变为 $\mathrm{MIP}$-时甲基 $\mathrm{C}$ 上获得负电荷 $(0.01895 e)$ 比 $\mathrm{a}, \mathrm{c}(0.01815 e, 0.01726 e)$ 取代时多, 使 得 MIP 的 2 位 $\mathrm{C}$ 原子形成相对于 MIP-更为缺电子 的状态, 利于 $\mathrm{O}_{2}^{--}$的进攻.

图 4 也表现了 MIP 阴离子的静态诱导效应较 强, 导致酮基氧、甲基碳、 $\mathrm{N} 7$ 位上和自由基 $\mathrm{C} 2$ 上的 电荷分布较多; MIP 自由基的共轭效应增强, 主要 表现为 $\mathrm{C}=\mathrm{O}$ 键长、取代基与 MIP 连接处的 $\mathrm{C}-\mathrm{C} 6$ 键长都变短. 这样, 从 MIP 阴离子到自由基过程中, 酮基氧、 $\mathrm{N} 7$ 和 $\mathrm{C} 2$ 位上的电荷数变化相对其他原子 要明显, 尤其是酮基氧变化更加明显(见图 4). 在气 相中从 MIP 阴离子到 MIP 自由基的过程中, MIPa- 


\begin{tabular}{llllll} 
& \multicolumn{2}{c}{ DG } & \multicolumn{2}{c}{ DMSO } \\
HOMO & LUMO & HOMO & LUMO & HOMO & LUMO
\end{tabular}
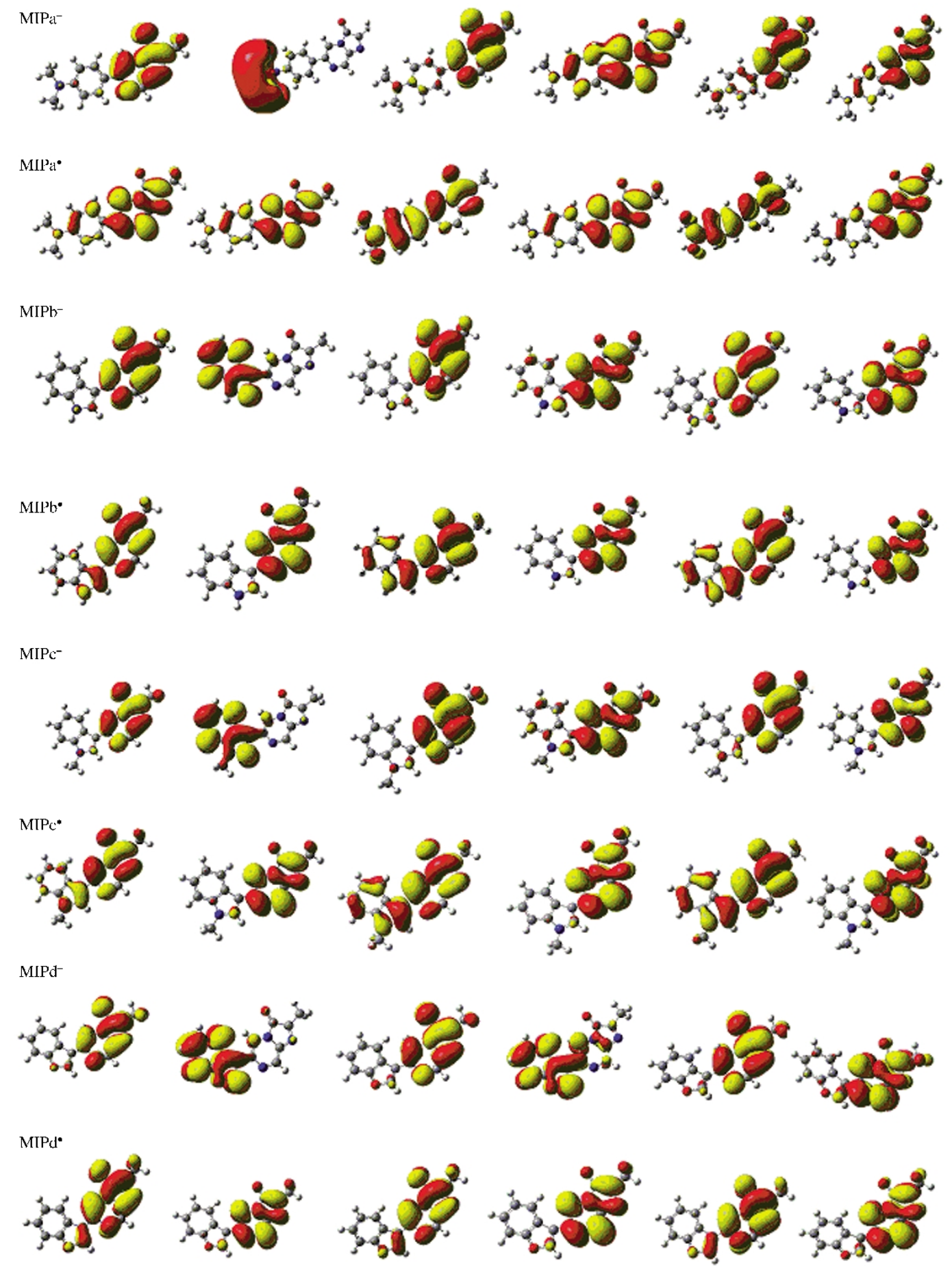

图 3 不同介质中 MIPa-MIPd 阴离子和自由基的相关前线分子轨道

Fig.3 Relevant frontier orbitals of the anions and free radicals for compound MIPa-MIPd in different media

$\mathrm{MIPc}$ 上 $\mathrm{C}=\mathrm{O}$ 键长没有变化, 但是在 $\mathrm{DG}$ 溶剂中时 就发现当吲哚作为 MIP 取代基团时 $\mathrm{C}=\mathrm{O}$ 键长相 对其他取代基团变化大很多, 而且 $b$ 取代相对于 $a$, $\mathrm{c}$ 取代时整个 MIP 片段的电荷转移数最大, 即 $\mathrm{b}$ 取 代基对母体的影响最大, 说明取代基为 $\mathrm{b}$ 时对这步
反应过程起到了积极的作用.

$\mathrm{MIP}^{-}$到 MIP・过程中咪唑并吡嗪酮环在 DG 溶 剂中的电荷分布差值分别为 $0.88872 e$ (a), $0.90799 e$

(b) 和 $0.90321 e(\mathrm{c})$. 在溶剂 $\mathrm{DG}$ 中进行 $\mathrm{MIP}^{-} \rightarrow \mathrm{MIP}^{\bullet}$ 的反应时, 中心片段咪唑并吡嗪酮环(母体)的电荷 

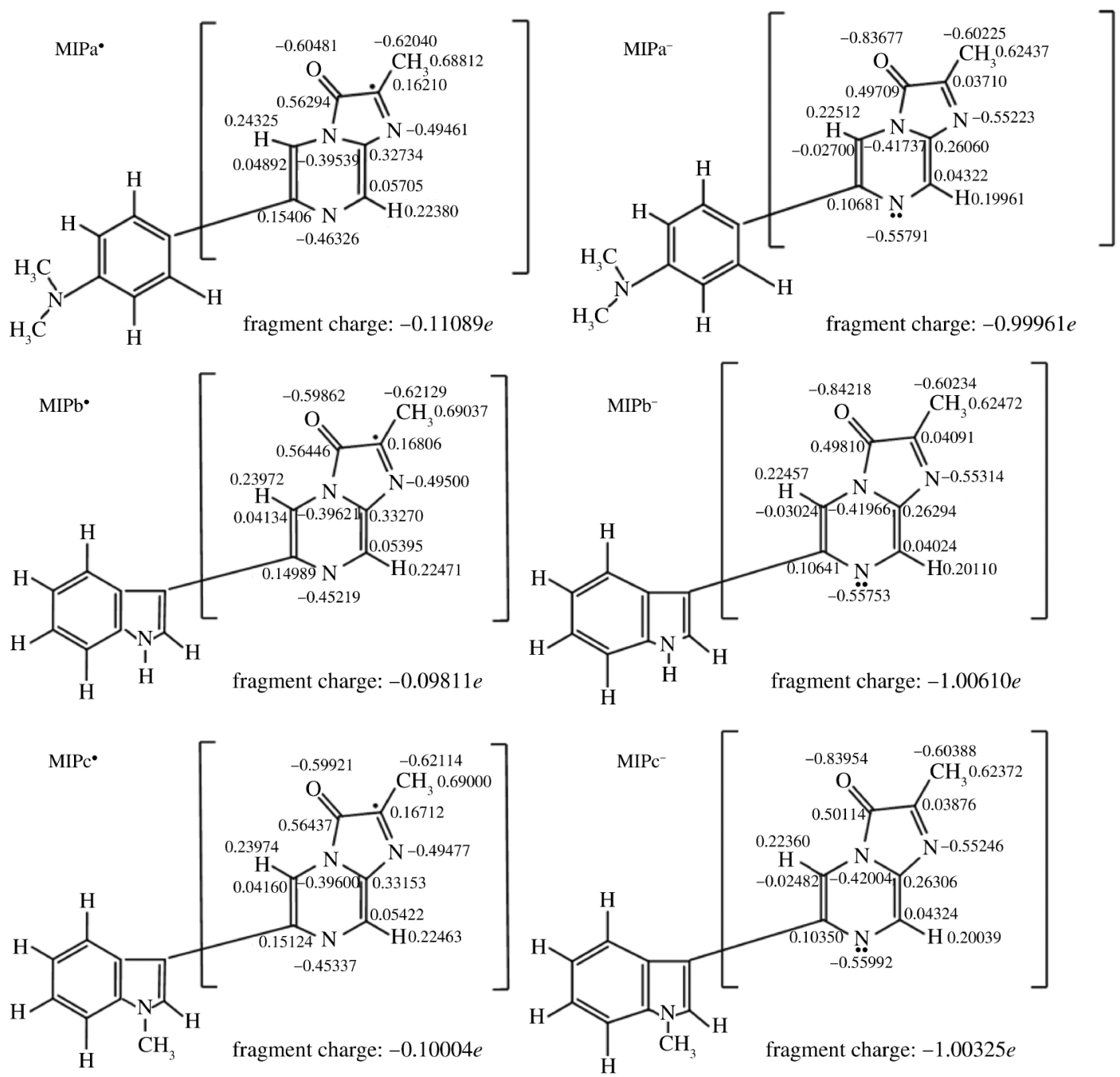

图 4 在 DG 中 MIPa-MIPc 阴离子和自由基的 NPA

Fig.4 NPA of the anions and free radicals of MIPa-MIPc in DG

The data beside atoms are charges in $e$.

差绝对值越大说明取代基给电子能力越强. 因此, $b$ 和 $\mathrm{a}, \mathrm{c}$ 相比是最好的给电子体. 这个结果与电子抽 取能的计算结论相一致. 在 $\mathrm{MIP}^{-} \rightarrow \mathrm{MIP} ・$ 过程中, 给 电子基团吲哚取代基与咪唑并吡嗪酮环相连时单电 子转移效率最大, 促使反应 $\mathrm{MIP}^{-} \rightarrow \mathrm{MIP} \cdot$ 快速进行.

\section{3 结 论}

从理论上研究了海萤衍生物即以咪唑并吡嗪酮 环为骨架, 在 C6 位上用不同的芳基进行取代形成 的一系列衍生物. 对其发光反应的关键步骤中涉及 的阴离子和自由基做了几何结构、键长变化、HOMOLUMO 能隙分析、电子抽取能的计算和自然电荷布 居分析. 通过阴离子和自由基中的 $\mathrm{C}-\mathrm{C} 6$ 以及 $\mathrm{C}=$ $\mathrm{O}$ 键长分析表明, 给电子基团与中心结构的 $\pi$ 共轭
相互作用都强于吸电子基团, 并且溶剂效应增强了 给电子基团与中心结构片段之间的相互作用. 在 DG 中 $\mathrm{MIPb}^{-}$的 HOMO-LUMO 能隙值变化比较大, 吲哚作为咪唑并吡嗪酮环芳基(C6)位取代基时电子 抽取能最小、阴离子和自由基之间的电荷差最大, 表 明在这种溶剂中其给电子能力增强、稳定性降低, 更 容易实现 $\mathrm{MIP}^{-} \rightarrow \mathrm{MIP} ・$ 这个过程, 这对提高海苂类 似物苂光效率起到了积极的作用. 我们将在接下来 的工作里研究海萤发光机理的全过程. 总之, 本研究 从理论计算上验证了在 DG 这种溶剂中, 咪唑并吡 嗪酮环 C6 位的取代基为吲哚时类似海萤的原型, 有较高的电荷转移效率和较低的 HOMO-LUMO 能 隙和电子抽取能, 增加海萤类似物发光反应过程的 速率, 有助于产生较高的发光效率. 


\section{References}

1 Shimomura, O.; Goto, T.; Hirata, Y. Chem. Soc. Jpn., 1957, 30 : 929

2 Yang, Y. B.; Ji, S. M.; Zhou, F. K.; Zhao, J. Z. Biosensors and Bioelectronics, 2009, 24: 3442

3 Wu, C.; Kawasaki, K. Anal. Chem., 2007, 79: 1634

4 Tan, D. J.; Gu, R. X. Food and Machinery, 1994, 3: 25 [谭道经, 顾瑞霞. 食品与机械, 1994, 3: 25]

5 Hu, Z.; Yin, J. G.; Jiang, N.; Chen, Q. J. Chin. J. Vet. Sci., 2008, 28: 732 [胡 哲, 尹继刚, 姜 宁, 陈启军. 中国兽医学报, 2008, 28: 732]

6 Lin, H. Envi. Sci., 1981, 6: 67 [林 海. 环境科学, 1981, 6: 67]

7 Yang, X. L. Continuing Medical Education, 2007, 21: 56 [杨晓 林. 继续医学教育, 2007, 21: 56]

8 Zhang, J. C.; Wang, D. Y. Modern photochemistry. Beijing: Beijing Industry Press, 2006: 319-320］张建成, 王夺元. 现代光 化学. 北京: 北京工业出版社, 2006: 319-320]

9 Katsunori, T. Bioorg. Chem., 2007, 35: 82

10 Shimomura, O.; Johnson, F. H.; Masugi, T. Nature, 1969, 164: 1299

11 Shimomura, O.; Johnson, F. H. Methods Enzymol., 1978, 57: 331

12 Kishi, Y.; Goto, T.; Hirata, Y.; Shimomura, O.; Johnson, F. H. Tetrahedron Lett., 1966, 7: 3427

13 Kishi, Y.; Goto, T.; Inoue, S.; Sugiura, S.; Kishimoto, H. Tetrahedron Lett., 1966, 7: 3445

14 Takahashi, Y.; Kondo, H.; Maki, S.; Niwa, H.; Hiroshi, I.; Takashi, H. Tetrahedron Lett., 2006, 47: 6057

15 Kondo, H.; Igarashi, T.; Maki, S.; Niwa, H.; Ikeda, H.; Hirano, T.
Tetrahedron Lett., 2005, 46: 7701

16 Hohenberg, P.; Kohn, W. Phys. Rev. B, 1964, 136: 864

17 Kohn, W.; Sham, L. J. Phys. Rev. A, 1965, 140: 1133

18 Becke, A. D. Phys. Rev. A, 1988, 38: 3098

19 Becke, A. D. J. Chem. Phys., 1993, 98: 5648

20 Lee, C.; Yang, W.; Parr, R. G. Phys. Rev. B, 1988, 37: 785

21 Navizet, I.; Liu, Y. J.; Ferre, N.; Xiao, H. Y.; Fang, W. H.; Lindh, R. J. Am. Chem. Soc., 2010, 132: 706

22 Min, C. G.; Ren, A. M.; Guo, J. F.; Li, Z. W.; Zou, L. Y.; Goddard, J. D.; Feng, J. K. ChemPhysChem, 2010, 11: 251

23 Frisch, M. J.; Trucks, G. W.; Schlegel, H. B.; et al. Gaussian 03. Revision B.01. Pittsburgh, PA: Gaussian Inc., 2003

24 Mennucci, B.; Tomasi, J. J. Chem. Phys., 1997, 106: 5151

25 Hirano, T.; Takahashi, Y.; Kondo, H.; Maki, S.; Kojima, S.; Hiroshi, I.; Haruki, N. Photochem. Photobiol. Sci., 2008, 7: 197

26 Okajima, T.; Tokuda, K.; Ohsaka, T. Bioelectrochem. Bioenerg., 1996, 41: 205

27 Goto, T. Pure Appl. Chem., 1968, 17: 421

28 Johnso, F. H.; Satchel, H. D.; Taylor, E. C.; Shimomura, O. Chemiluminescence and fluorescence of cypridina luciferin and of some new indole compounds in dimethylsufoxide//Bioluminescence in Progress. Princeton: Princeton University Press, 1966: 67

29 Goto, T.; Inoue, S.; Sugiura, S.; Nishikawa, K.; Isobe, M.; Abe, Y. Tetrahedron Lett., 1968, 37: 4035

30 Toya, Y.; Kayano, T.; Sato, K.; Goto, Y. Chem. Soc. Jpn., 1992, 65: 2475

31 Nakamura, H.; Aizawa, M.; Takeuchi, D.; Murai, A.; Shimomura O. Tetrahedron Lett., 2000, 41: 2185 